\title{
Simposios Ibero-latinoamericanos de Terminología Morfológica (SILAT). Primeros Dos Años y Normativas Vigentes
}

\author{
Iberia-Latin-American Symposia of Morphological Terminology (SILAT). \\ First Two Years and Statute
}

"Ricardo Jorge Losardo; ** Rolando Cruz Gutiérrez; ${ }^{* * * *}$ Alberto Rodríguez Torres; *****José Carlos Prates; \& ******Nadir Eunice Valverde Barbato de Prates

LOSARDO, R. J.; CRUZ, G. R.; RODRÍGUEZ, T. A.; PRATES, J. C.; \& PRATES, N. E. V. B. Simposios Ibero-latinoamericanos de terminología morfológica (SILAT). Primeros dos años y normativas vigentes. Int. J. Morphol., 28(4):1323-1326, 2010.

RESUMEN: Con motivo de cumplir los dos primeros años de vida, se realiza una reseña de SILAT como programa científico y educativo que sirve de instrumento para analizar y solucionar los problemas de la terminología médica morfológica en los países de habla hispana y portuguesa de América. Se describen los fundamentos de su creación, su estrategia y el alcance en la región, los años fundacionales y su futuro inmediato. Finalmente, se señalan algunas conclusiones y se anexa su Estatuto.

PALABRAS CLAVE: Simposios Ibero-latinoamericanos; Terminología Anatómica, Histológica y Embriológica; Morfología.

\section{FUNDAMENTO}

SILAT es una entidad científica-educativa, sin fines de lucro, que se centra en el estudio de los términos médicos en el área de la morfología y que abarca la anatomía, la histología y la embriología. Tiene el objeto de lograr precisión y claridad en las denominaciones de las estructuras del cuerpo humano.

Constituye una herramienta que permite resolver cuestiones terminológicas y ayuda a mejorar la educación médica en la región.

El SILAT fue un objetivo de la Asociación Panamericana de Anatomía (APA) -por iniciativa de su Comisión Directiva y el apoyo de sus presidentes honorarios activospara promover la participación en esta problemática en todos los países hispano-luso parlantes, elevando el compromiso de la región.

En síntesis: SILAT estudia, analiza, traduce y divulga la terminología morfológica internacional con el fin que las instituciones educativas de medicina y de otras áreas de la salud, de habla hispana y portuguesa, la empleen cotidianamente.

\section{Estrategia. Alcances.}

SILAT cuenta con la adhesión de APA, institución de más de 40 años de vida y con una extensa tradición académica en las ciencias morfológicas.

Utiliza un modelo centrado en la participación de todos los profesores e investigadores universitarios de la región, interesados y comprometidos con la temática.

Las actividades de SILAT se llevan a cabo en las universidades y se articulan con las sociedades científicas.

Busca lograr la unificación de las denominaciones de las estructuras anatómicas, histológicas y embriológicas que en la práctica aún no se han conseguido, con el fin de

\footnotetext{
Presidente Honorario de Asociación Panamericana de Anatomía. Facultad de Medicina, Universidad del Salvador (USAL), Buenos Aires, Argentina. ** Presidente de Asociación Panamericana de Anatomía. Miembro de FICAT. Facultad de Medicina, Universidad de Costa Rica (UCR), San José, Costa Rica.

**** Presidente Honorario de Asociación Panamericana de Anatomía. Facultad de Medicina, Universidad de Chile, Santiago, Chile.

${ }^{* * * *}$ Presidente Honorario de Asociación Panamericana de Anatomía. Ex miembro de FICAT. Universidade Federal de Sao Paulo (UNIFESP), Sao Paulo, Brasil.

${ }^{* * * * *}$ Ex-Secretaria General de Asociación Panamericana de Anatomía. Instituto de Ciencias Biomedicas (ICB). Universidade de Sao Paulo (USP), Sao Paulo, Brasil.
} 
alcanzar el uso de un lenguaje científico común facilitando el aprendizaje y la comunicación en Ibero-América.

Los expertos que integran el SILAT se reúnen durante varias jornadas completas de trabajo, estudiando y discutiendo problemas terminológicos tanto internacionales como regionales.

SILAT promueve un cambio académico en la región; y permite la participación y expresión de universitarios en un ámbito científico hasta entonces inexistente. Por ello, la terminología morfológica internacional debe trasladarse a los programas de la enseñanza universitaria en esta región.

Estamos convencidos que la incorporación paulatina y progresiva de estos cambios serán fruto del trabajo mancomunado de SILAT, las universidades, las sociedades científicas, los ministerios de educación y salud, etc. Ello permitiría optimizar los recursos disponibles para que los resultados tengan un alto impacto. Por otro lado, esto expresaría un compromiso a la hora de planear políticas públicas y la preocupación de avanzar en logros educativos y científicos en cada uno de los países intervinientes.

Los dos primeros años. En estos primeros dos años, fundacionales, se realizaron cinco SILAT y se puso en marcha algo nuevo, hasta hace poco inexistente e impensable.

Estos dos años, que se iniciaron en el 2009 con el Acta Fundacional en San José de Costa Rica y que en el 2010 terminaron con la aprobación del Estatuto en San Pablo (Brasil) y culminaron con la realización de un SILAT en un Congreso Panamericano de Anatomía en Temuco (Chile), permiten considerar a este período como "fundacional".

En estos foros se reúnen periódicamente morfólogos de toda la región, constituyéndose en su recurso más sensible y relevante. Estuvieron financiadas por las universidades- sedes con aportes únicos y aislados. La suma de ambos esfuerzos -recursos humanos y financieros- logrados en forma continúa y sostenida permitieron el resultado actual.

Expertos de quince países estuvieron presentes en estos cinco foros:

a) Sub-región América del Norte: EE.UU. y México

b) Sub-región América Central y Caribe: Costa Rica, El Salvador, Guatemala, Honduras y Nicaragua.

c) Sub-región América del Sur: Argentina, Bolivia, Brasil, Chile, Colombia, Ecuador, Perú y Venezuela.

Hoy día, nos encontramos coordinando los últimos detalles para poner en funcionamiento los SILAT 2011, fortaleciéndolo e incorporando otros países y miembros.

\section{Lista de SILAT realizados. Fecha, sedes, universidades y presidentes.}

I. 13-16 abril 2009: San José, Costa Rica. Universidad de Costa Rica. Prof. Dr. Rolando Cruz Gutiérrez.

II.15-18 septiembre 2009: Lima, Perú. Universidad San Martín de Porres. Prof. Dr. Germán Molina Albornoz.

III. 26-30 abril 2010: San José, Costa Rica. Universidad de Costa Rica. Prof. Dr. Rolando Cruz Gutiérrez.

IV. 03-07 octubre 2010: San Pablo, Brasil. Universidad de San Pablo. Prof. Dra. Nadir Eunice Valverde Barbato de Prates.

V. 25-26 octubre 2010: Temuco, Chile. Universidad de la Frontera. Prof. Dr. Alberto Rodríguez Torres.

El futuro inmediato. SILAT se debe consolidar como un instrumento adecuado y organizado que busca satisfacer necesidades y brindar respuestas superadoras a los problemas de la terminología médica morfológica.

En las puertas de esta segunda década del siglo XXI el desafío será fortalecer el SILAT. Para ello, se deberán llevar acciones acordes con la época de globalización en que se vive.

La región ibero-latinoamericana debe abordar hoy esta problemática científica y educativa, en un marco participativo e integrado, con todos los países que componen la región sin exclusión alguna.

Se debe dar una larga y dura batalla para la solución de estas cuestiones terminológicas, propias de una segmentación y fragmentación de nuestras ciencias médicas y biológicas en un ámbito geográfico muy extenso.

\section{CONCLUSIONES}

1. SILAT destaca las ciencias morfológicas como base de las ciencias médicas.

2. Es un emprendimiento científico-educativo que por sus fines, sus objetivos y su dinámica, es trascendente en el contexto ibero-latinoamericano.

3. Está llevando adelante el compromiso de trabajar en una construcción colectiva de la terminología morfológica internacional en pos de una mejor calidad de educación y de ciencia.

4. Los resultados se reflejarán en el campo de la investigación, la docencia y la práctica médica asistencial a nivel ibero-latinoamericano, facilitando el intercambio científico y el progreso en las ciencias médicas. 


\section{ESTATUTO DEL SIMPOSIO IBERO-LATINOAMERICANO DE TERMINOLOGÍA (SILAT)}

\section{CAPÍTULO I . Nombre}

Artículo 1.- Se denominará "Simposio Ibero-latinoamericano de Terminología" (SILAT), precedido de un número romano y en orden progresivo (I, II, III, etc.).

\section{CAPÍTULO II . Objetivos y finalidades}

Artículo 2.- Los Simposios Ibero-latinoamericanos de Terminología tienen como objetivo y finalidad:

1. Estimular y fomentar el conocimiento de la terminología morfológica internacional a través de simposios, seminarios, jornadas, cursos, mesas redondas, conferencias, publicaciones, etc. en las universidades de los países de habla hispana y portuguesa.

2. Estudiar y analizar las traducciones en español y portugués de la terminología morfológica internacional.

3. Promover capacitación y brindar asesoramiento sobre terminología morfológica internacional a los docentes e investigadores universitarios ibero-latinoamericanos.

4. Desarrollar y promover investigaciones en las universidades de los países ibero-latinoamericanos, orientadas al análisis de los problemas de la terminología anatómica, histológica y/o embriológica y sus posibles soluciones.

5. Asesorar a los países miembros en el estudio e implementación de reformas en los programas tendientes a modernizar la enseñanza universitaria en el campo de la terminología morfológica internacional.

6. Sustentar, como finalidad primordial, la integración regional a la terminología morfológica internacional vigente.

\section{CAPÍTULO III. Periodicidad}

Artículo 3.- a) El Simposio se realizará al menos una vez por año y este plazo podrá ser menor de acuerdo con las conveniencias.

b) Las actividades del SILAT pueden adherirse a un Congreso Panamericano, a un Simposio internacional de ciencias morfológicas en América y/o península Ibérica o a un evento universitario de estas regiones, que ofrezca ser anfitrión.

c) En todos los casos, habrá coordinación con las Comisiones Directivas de la Asociación Panamericana de Anatomía y de la Academia Panamericana de Anatomía.

\section{CAPÍTULO IV . Sede y duración}

Artículo 4.- La sede del Simposio rotará de un evento a otro dentro de la región ibero-latinoamericana.

Artículo 5.- La duración del Simposio será de acuerdo a las posibilidades de cada país y su extensión no deberá superar los 5 días.

\section{CAPÍTULO V . Actividades}

Artículo 6.- Las actividades del SILAT comprenderán:

1. Promoción: coordinar y promover los esfuerzos para la divulgación y utilización de la terminología médica internacional en las universidades ibero-latinoamericanas.

2. Docencia: adiestramiento y capacitación de docentes en esta materia (en especial del sector público) a través de simposios, seminarios, jornadas, cursos, etc. Una función básica de SILAT es la formación de instructores con el fin de lograr un efecto multiplicador. Preparación de cuadros técnicos, capaces de llevar a cabo -en el ámbito nacional- tareas de adiestramiento en las áreas específicas (anatomía, embriología e histología) para las que han sido capacitados.

3. Investigación: estudios -tanto a nivel regional como nacionalque sirven de base para la búsqueda de un lenguaje científico común para toda la comunidad ibero-latinoamericana de las ciencias morfológicas. Formulación de propuestas de terminologías regionales y nacionales, puestas a consideración y discusión en el Comité de Expertos de SILAT.

4. Publicaciones: llevar a cabo una labor de documentación en el campo científico y educativo; y realizar una serie de publicaciones: a) informes de simposios, seminarios, jornadas, conferencias, mesas redondas; y b) folletos, boletines, cuadernillos y libros sobre terminología anatómica, histológica y embriológica.

5. Asesoría técnica: proyectos de asistencia y cooperación técnica en la materia a las universidades ibero-latinoamericanas y organismos que lo soliciten.

\section{CAPÍTULO VI. Comité de Expertos}

Artículo 7.- El Comité de Expertos tendrá las siguientes categorías:

a) Miembros Expertos. Aquellos profesionales -docentes y/o investigadores- que por sus méritos en el campo de la terminología estén en condiciones de dar asesoramiento, asistencia, adiestramiento y capacitación a terceros en la materia y dirigir proyectos sobre el tema.

b) Miembros Colaboradores. Aquellos profesionales que colaboren con las tareas que realizan los Expertos.

\section{CAPÍTULO VII. Gobierno}

Artículo 8.- El Gobierno del SILAT estará constituido por el Comité de Expertos, el Consejo Directivo y el Comité Organizador Local del evento.

Artículo 9.- El Consejo Directivo estará integrado por todos los Presidentes Honorarios de la Asociación Panamericana de Anatomía y los Ex Presidentes de los Comités Organizadores Locales de los SILAT.

Artículo 10.- El Comité Organizador Local, nombrado por su Pre- 
sidente, será el responsable de organizar el Simposio en la sede seleccionada.

Artículo 11.- La designación del país-sede será a solicitud de una propuesta elevada por un socio de la APA y apoyada por un Presidente Honorario de la APA al Cuerpo Directivo.

Artículo 12.- La sede y el Presidente próximo serán seleccionados entre los miembros de los países asistentes al último Simposio.

\section{CAPÍTULO VIII. Inscripciones}

Artículo 13.- El Comité Organizador Local del Simposio está ampliamente facultado para supervisar las inscripciones, que serán individuales y aceptarlas o rechazarlas según esté justificado.

Artículo 14.- Aquellas personas que no integren el Comité de Expertos y que por sus actividades estén interesados en concurrir y que deseen asistir solamente a las Sesiones de determinado tema en particular, se inscribirán como "Miembros Asistentes" al SILAT.

\section{CAPÍTULO IX. Reuniones de gobierno}

Artículo 15.- El Gobierno del SILAT tendrá una sesión administrativa durante el Simposio que se llevará a cabo con el siguiente orden del día:

a) Informes de actividades realizadas.

b) Elección de sede y Presidente del siguiente Simposio.

c) Otros asuntos relacionados con el gobierno y el desarrollo de los Simposios.

\section{CAPÍTULO X. Continuidad}

Artículo 16.- El Consejo Directivo de SILAT será responsable de asegurar la continuidad de los Simposios.

\section{CAPÍTULO XI. Publicaciones}

Artículo 17.- El Comité Organizador Local se comprometerá a publicar el programa de las actividades del Simposio, que serán distribuidas entre los asistentes al evento.

Artículo 18.- Los resúmenes de las comunicaciones científicas y conferencias del SILAT serán publicados en algunos de los órganos oficiales de la Asociación Panamericana deAnatomía.

Artículo 19.- Se publicará un Directorio con los miembros del SILAT.

Artículo 20.- Las actividades de prensa y difusión del Simposio serán responsabilidad del Comité Organizador Local y del Consejo Directivo de SILAT.

\section{CAPÍTULO XII. Modificaciones al Estatuto}

Artículo 21.- Las proposiciones concretas de modificaciones deberán presentarse por escrito, avaladas por lo menos por un Presidente Honorario de la APA y dirigidas al Consejo Directivo del SILAT. Se presentarán para su discusión y votación en la sesión administrativa durante el Simposio. Su aprobación será por mayoría absoluta de votos.

Nota: Redactado por el Comité Ejecutivo y el Consejo Directivo de la Asociación Panamericana de Anatomía. Aprobado por los participantes durante el desarrollo del IV SILAT, el 4 de octubre de 2010, en el centro de Convenciones Rebouças, São Paulo, Brasil.

LOSARDO, R. J.; CRUZ, G. R.; RODRÍGUEZ, T. A.; PRATES, J. C.; \& PRATES, N. E. V. B. Iberia-Latin-American Symposia of Morphological Terminology (SILAT). First Two Years and Statute. Int. J. Morphol., 28(4):1323-1326, 2010.

SUMMARY: On the occasion of meeting the first two years of life, it makes a review of SILAT as scientific and educational program that serves as a tool to analyze and solve the problems of morphological medical terminology in the countries of Hispanic and Portuguese-speaking America. It describes the basis of its creation, strategy and scope in the region, the founding years and its immediate future. Finally, some conclusions are indicated and it Statute is annexed.

KEY WORDS: Iberia-Latin-American Symposia; Anatomical, Histological and Embryological Terminology. Morphology.

\section{REFERENCIAS BIBLIOGRÁFICAS}

Cruz Gutiérrez, R.; Rodríguez Torres, A.; Prates, J. C.; Losardo, R. J.; Valverde Barbato, N. E. Simposio Ibero-latinoamericano de Terminología Morfológica. Características Generales. Int. J. Morphol., 28(2):643-6, 2010.

Cruz Gutiérrez, R.; Rodríguez Torres, A.; Prates, J. C.; Losardo, R. J.; Valverde Barbato, N. E. Simposios Ibero Latinoamericanos de Terminología. Anatomía, Histología y Embriología. Int. J. Morphol., 28(1):333-6, 2010.

Losardo, R. J. Asociación Panamericana de Anatomía: Reseña histórica y normativas vigentes. Int. J. Morphol., 27 (4):1345-52, 2009.

Dirección para correspondencia

Prof. Dr. Ricardo Jorge Losardo

Lavalle 1844 - Piso $5^{\circ}$ - Oficina 30

(1051) Ciudad de Buenos Aires

ARGENTINA

Tel./ Fax.: 0054-11-4372-4604

Email: ricardo.losardo@salvador.edu.ar 